
\title{
BReserch Soutere \\ Experimental study of electromechanical measurement, surface analysis and corrosion inhibition of mild steel in 1M HCL by Schiff's bases (SBs)
}

Dr. Bangarappa L ( $\sim$ bangaru10388@gmail.com )

University Visvesvaraya College of Engineering

\section{Research Article}

Keywords: Scanning Electron Microscopy (SEM), Energy-Dispersive X-ray Spectroscopy (EDX), mild steel surface in $1 \mathrm{M} \mathrm{HCL}$, Electromechanical Impedance Spectroscopy (EIS), three Schiff's bases (SBs)

Posted Date: January 7th, 2022

DOI: https://doi.org/10.21203/rs.3.rs-1203911/v1

License: (c) (1) This work is licensed under a Creative Commons Attribution 4.0 International License. Read Full License 


\title{
Experimental study of electromechanical measurement, surface analysis and corrosion inhibition of mild steel in 1M HCL by Schiff's bases (SBs)
}

\author{
Dr. Bangarappa $L^{*}$ \\ *Prof., Mechanical Engineering Department, University Visvesvaraya College of Engineering, \\ Bengaluru, Karnataka, India
}

\begin{abstract}
:
Potentiodynamic polarization measurements indicate that SBs acts as mixed type corrosion inhibitors. the morphology of the mild steel surface is investigated by scanning electron microscopy (SEM) and the surface composition was evaluated using energydispersive X-ray Spectroscopy (EDX) to show the presence of SBs on the mild steel surface in 1M HCL. The present study, three Schiff's bases (SBs) namely 2 (2hydroxybenzylideneamino) heptanedioic acids, 2 (4-dimethylamino benzlideamino) heptanedioic acids and 2 (4hydroxy-3-methoxybenzylideneamino) heptanedioic acids were synthesized. Using weight loss, potentiodynamic polarization and electromechanical impedance spectroscopy (EIS) techniques for corrosion inhibition properties on mild steel in 1M HCL has been investigated. The adsorption of SBs on the mild steel surface contains Langmuir adsorption isotherm. Here kinetic and thermodynamic parameters also determined to describe the mechanism of adsorption in relevance. The main object of this presentation is experimental study of the inhibiting action of synthesized Schiff's bases of aldehydes containing nitrogen, oxygen and aromatic rings and Glutamic acid.
\end{abstract}

Keywords: Scanning Electron Microscopy (SEM), Energy-Dispersive X-ray Spectroscopy (EDX), mild steel surface in 1M HCL, Electromechanical Impedance Spectroscopy (EIS), three Schiff's bases (SBs).

\section{Introduction:}

The purpose of this research is to investigate the inhibitory effects of synthetic Glutamic acid Schiff bases and aldehydes comprising nitrogen, oxygen, and aromatic rings. Weight loss, potentiodynamic polarisation, and electrochemical impedance spectroscopy were used in the research (EIS) [1]. Several isotherms were also examined to see if they could accurately characterise the adsorption behaviour of the SBs under investigation. Weight loss experiments were carried out at various temperatures in the absence and presence of the optimum concentration of SBs to investigate the effect of temperature on mild steel corrosion behaviour [2].

The use of inhibitors to control the harmful effects of acid environments has become commonplace. Acid cleaning, acid pickling, acid descaling, and oil well acidizing are only a few examples of industrial operations. Because of its tremendous value, Mild steel has a wide range of applications in numerous industries as a construction material due to its mechanical qualities and low cost [3]. Chemical reactors, heat exchange and boiler systems, storage tanks, and oil and gas transportation pipes are all examples of this type of equipment. Compounds of organic origin Corrosion inhibitors with polar functionalities with oxygen, 
nitrogen, or sulphur atoms have been researched extensively. Some Schiff bases have recently been identified as effective mild steel corrosion inhibitors. Adsorption on a metal surface occurs, with polar groups acting as the adsorption centre [4]. The effective, existence of the -electron of the aromatic system and multiple bonds, or the presence of the -electron of the aromatic system and multiple bonds, causes adsorption and the inhibitor's molecular structure contains electronegative atoms (O or N) [5].

\section{Experimental setup \\ 2.1 Inhibitors synthesis}

The SBs were made using the scheme 1 as a guide. Sigma Aldrich Pvt. Ltd provided all of the chemicals and solvents required for the synthesis (India). In a typical procedure, ethanolic aldehydes solution is combined with alkaline ethanolic glutamic acid solution and stirred for 30 minutes. The completed mixture is stirred constantly for around 15 minutes. TLC was used to monitor the reaction's conclusion. Filtered, washed with cold ethanol, and dried were the final steps [6]. The synthesis was also carried out using ultrasonic techniques, with the same reaction mixture being sonicated in an ultrasonic bath for 20 minutes. Thinlayer chromatography with ethyl acetate/nhexane (4:6) as the developing solvent and the Silica Plate (TLC PlatesAluminum (Al) Silica proved the purity of the product [7].

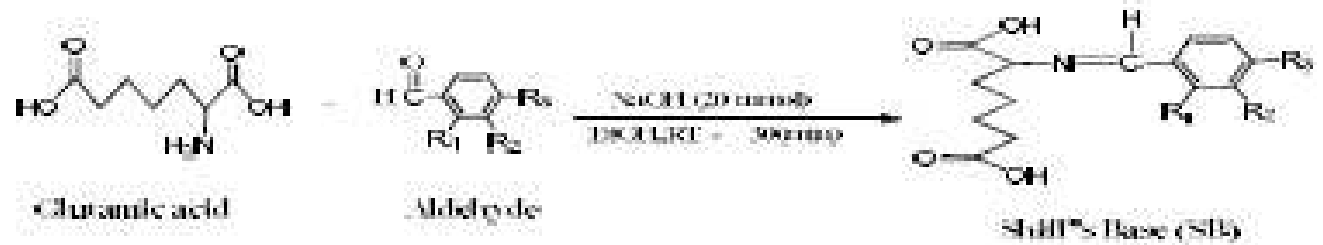

Figure1 Schematic bases for synthesis of HCL

\subsection{Mild steel materials}

Mild steel specimens with the following compositions (wt\%): Fe 99.30 percent, C 0.076 percent, Si 0.026 percent, Mn 0.192 percent, $\mathrm{P} 0.012$ percent, $\mathrm{Cr} 0.050$ percent, $\mathrm{Ni}$ 0.050 percent, $\mathrm{Al} 0.023$ percent, and $\mathrm{Cu} 0.135$ percent were successively abraded with emery papers ranging from 600 to $1200 \mathrm{mesh} / \mathrm{in}$ grade. The mild steel specimen was cleaned in double distilled water, degreased with acetone, and dried using a hot air blower. The working electrode (WE) was a $7.0 \mathrm{~cm}$ long stem (separated with epoxy resin) with a surface area of $1.0 \mathrm{~cm}^{2}$ for electrochemical measurements and a volume of $2.52 .00 .025 \mathrm{~cm}^{3}$ for weight loss studies [8]. Analytical reagent grade reagent (37 percent HCL) and double distilled water were used to make the test solution of $1 \mathrm{M} \mathrm{HCL}$.

Table 1 Aldehyde aromatic rings have a variety of substituents

\begin{tabular}{|l|l|l|l|}
\hline Schiff's Base & $\mathrm{R}_{1}$ & $\mathrm{R}_{2}$ & $\mathrm{R}_{3}$ \\
\hline SB-1 & $-\mathrm{OH}$ & $-\mathrm{H}$ & $-\mathrm{H}$ \\
\hline SB-2 & $-\mathrm{H}$ & $-\mathrm{H}$ & $-\mathrm{N}^{\left.-\mathrm{CH}_{3}\right)_{2}}$ \\
\hline SB-3 & $-\mathrm{H}$ & $-\mathrm{OH}$ & $-\mathrm{OCH}_{3}$ \\
\hline
\end{tabular}

\subsection{Weight loss method}

As previously stated, the weight reduction measures were taken using a recognised procedure. The inhibition efficiency ( $\eta$ percentage) and surface coverage $(\Theta)$ were determined using the following equations: where CR and CR (i) are the corrosion rate values in the absence and presence of SBs, respectively. The mild steel corrosion rate (CR) in acidic 
medium was determined using the following equation: where $\mathrm{W}$ is the weight loss of mild steel specimens $(\mathrm{mg}), \mathrm{A}$ is the area of the specimen $\left(\mathrm{cm}^{2}\right)$, and $\mathrm{t}$ is the exposure period $(\mathrm{h})$.

$$
\begin{array}{ll}
\eta \%=\frac{C_{\mathrm{R}}-C_{\mathrm{R}(i)} \times 100}{C_{\mathrm{R}} \times 100} & C_{\mathrm{R}}=\frac{W}{A t} \\
\theta=\frac{C_{\mathrm{R}}-C_{\mathrm{R}(i)}}{C_{\mathrm{R}}} &
\end{array}
$$

\subsection{Electromechanical measurements}

All electrochemical measurements were performed with a Potentiostat/Galvanostat with a Gamry framework system based on ESA 400 in a frequency range of 10-2 Hz to 105 $\mathrm{Hz}$ under Potentiodynamic circumstances, with amplitude of $10 \mathrm{mV}$ peak-to-peak, utilising AC signal at Ecorr. This Potentiostat/Galvanostat is made up of three electrode assemblies: mild steel for the working electrode, saturated calomel for the reference electrode, and platinum foil for the counter electrode. Software DC105 for corrosion and EIS 300 for EIS measurements, as well as Echem Analyst version 5.50 software packages for data fitting, are all Gamry applications [9-12]. Aerated solutions were used for the electrochemical tests. The working electrode was submerged in $1 \mathrm{M} \mathrm{HCl}$ in the absence and presence of SBs for 30 minutes prior to electrochemical experiments to stabilise the OCP w.r.t. SCE. All electrochemical impendence experiments were carried out in a potentiodynamic condition with amplitude of $10 \mathrm{mV}$ from $100,000 \mathrm{~Hz}$ to $0.01 \mathrm{~Hz}$.

$$
\begin{aligned}
& \eta \%=\left(1-\frac{I_{\text {corr }(\mathrm{i})}}{I_{\text {corr }}}\right) \times 100 \\
& \eta \%=\left(1-\frac{R_{a}}{R_{c e(0)}}\right) \times 100
\end{aligned}
$$

\section{Results and discussion}

\subsection{Wight loss studies}

\subsubsection{Effect of inhibitor concentration}

Table 2 shows the percentage inhibition efficiency (percent) and corrosion rate (CR) obtained from the weight loss method at various SB doses. The table shows that when SBs concentrations rise, inhibition efficiency rises. At $400 \mathrm{ppm}$, the maximum inhibitory efficiency was achieved, and additional increases in concentration had no discernible effect on the performance of SBs, implying that $400 \mathrm{ppm}$ was the optimum concentration. With increasing SBs concentrations, the value of corrosion rate drops and inhibition efficiency rises. This pattern could be explained by the fact that when SBs concentrations rose, so did adsorption and surface coverage [13-16]. Figure 2 depicts the fluctuation in inhibition efficiency as inhibitor concentrations increase (a). Table 2 shows that the inhibitory efficiency of SBs is in the following order: SB-1, SB-2, and SB-3. 


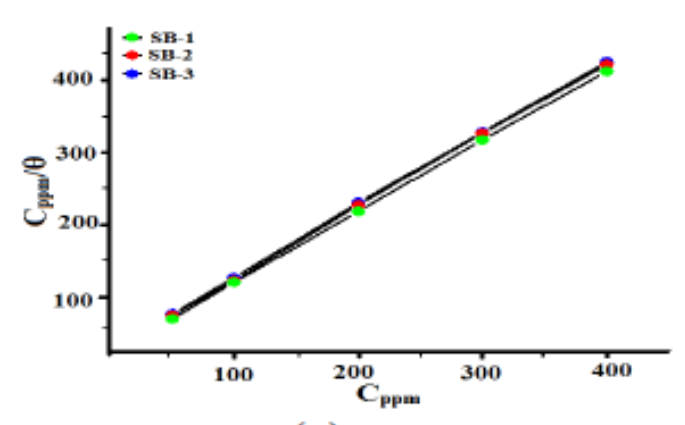

(a)

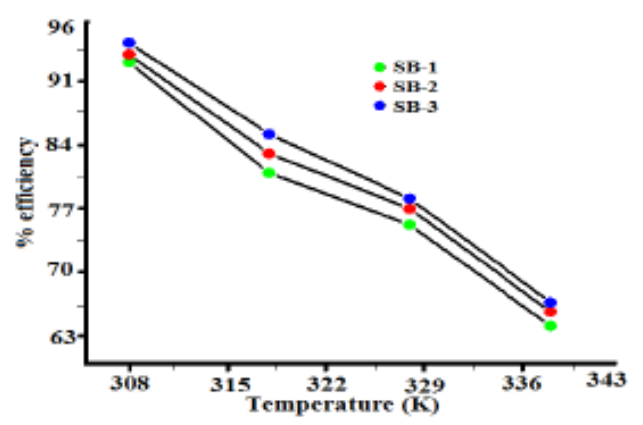

(b)

Figure: 2. a) Different concentration of SBs surface

b) Different Temperature of SBs Inhibition efficiency

\subsubsection{Effect of temperature}

Figure 2 (a) depicts the relationship between inhibition efficiency and solution temperature (b). It has been discovered that when temperature rises, inhibition efficiency diminishes. At higher temperatures, adsorption SBs molecules desorb from the metal surface, resulting in a decrease in inhibitory efficiency. More desorption of SBs occurs at higher temperatures, and a larger surface area of metal comes into touch with the acid environment, resulting in an increase in corrosion rate. These findings show that all SBs are effective inhibitors of mild steel corrosion in $1 \mathrm{M} \mathrm{HCl}$ over the temperature range examined [17-20]. The remarkable performance of the SBs under investigation is attributed to a strong contact between the mild steel surface and the molecules $(\Theta)$ of the SBs.

Table No. 2 Weight loss characteristics for mild steel in $1 \mathrm{M} \mathrm{HCl}$ in the absence and presence of different concentrations of SBs, such as Corrosion rate (CR), Surface coverage, and Corrosion inhibition (percent).

\begin{tabular}{|l|c|c|c|c|}
\hline Inhibitor & $\begin{array}{c}\text { Inhibitor conc } \\
\mathrm{Ppm}\end{array}$ & $\begin{array}{c}\text { Corrosion rate } \\
\left(\mathrm{mg} \mathrm{cm}^{-2} \mathrm{~h}^{-3}\right)\end{array}$ & $\begin{array}{c}\text { Surface coverage } \\
(\theta)\end{array}$ & $\mathbf{\eta} \mathbf{0}$ \\
\hline Blank & 0.0 & 85.33 & $\ldots \ldots$ & $\ldots$ \\
\hline \multirow{4}{*}{ SB-1 } & 50 & 30.05 & 0.6478 & 64.78 \\
\cline { 2 - 5 } & 100 & 18.18 & 0.7869 & 78.69 \\
\cline { 2 - 5 } & 200 & 11.50 & 0.8652 & 86.52 \\
\cline { 2 - 5 } & 300 & 7.92 & 0.9130 & 91.30 \\
\cline { 2 - 5 } & 400 & 9.19 & 0.9391 & 93.91 \\
\cline { 2 - 5 } & 50 & 28.56 & 0.6652 & 66.52 \\
\cline { 2 - 5 } & 100 & 15.95 & 0.8130 & 81.30 \\
\cline { 2 - 5 } & 200 & 10.38 & 0.8782 & 91.73 \\
\cline { 2 - 5 } & 300 & 7.04 & 0.9173 & 94.78 \\
\hline \multirow{4}{*}{ SB-2 } & 400 & 4.45 & 0.9478 & 81.30 \\
\cline { 2 - 5 } & 50 & 24.48 & 0.7130 & 91.30 \\
\cline { 2 - 5 } & 100 & 15.21 & 0.8270 & 94.34 \\
\cline { 2 - 5 } & 200 & 7.42 & 0.9130 & 96.95 \\
\cline { 2 - 5 } & 300 & 4.82 & 0.9695 & \\
\hline
\end{tabular}

\subsubsection{Thermodynamic parameters and adsorption isotherm}

On the basis of adsorption behaviour, the mechanism of corrosion inhibition may be elucidated. To explain the adsorption behaviour of SBs, several adsorption isotherms were examined, with the Langmuir adsorption isotherm providing the best fit. As demonstrated in Figure 3, the plot of $\log \mathrm{C}$ vs $\log \Theta(1-\Theta)$ yields straight lines for all SBs (a). Weight loss data was used to determine the degree of surface coverage $(\Theta)$ for varied concentrations of all SBs. 

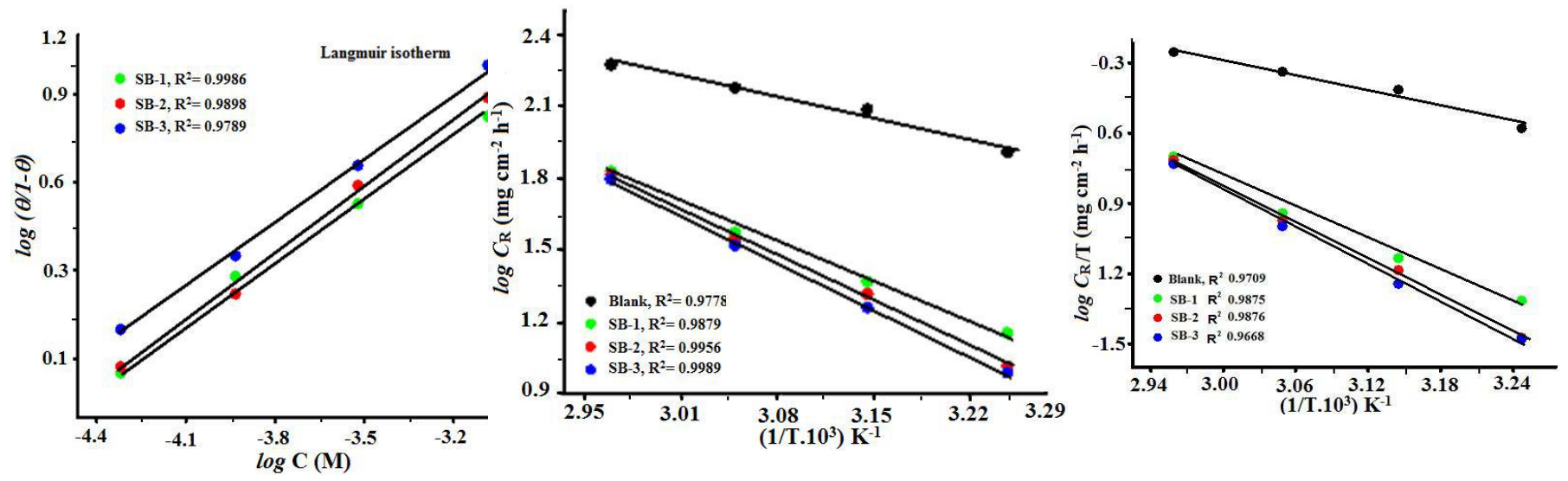

Figure 3 (a) Langmuir adsorption isotherm (b) Arrhenius plot of log CR Vs 1/T (c) Arrhenius plot of $\log C R / T$ Vs $1 / T$

Table 3: Thermodynamic parameters for mild steel in $1 \mathrm{M} \mathrm{HCl}$ in the absence and presence of the investigated SBs' optimal concentration

\begin{tabular}{|l|l|l|l|l|}
\hline Inhibitor & $E_{\mathrm{a}}\left(\mathrm{kJ} \mathrm{mol}^{-1}\right)$ & $\Delta H^{*}\left(\mathrm{~kJ} \mathrm{~mol}^{-1}\right)$ & $\Delta S^{*}\left(\mathrm{~J} \mathrm{~K}^{-1} \mathrm{~mol}^{-1}\right)$ & $\Delta G^{0}\left(\mathrm{~kJ} \mathrm{~mol}^{-1}\right)$ \\
\hline Blank & 28.48 & 26.04 & -148.9 & $\ldots \ldots \ldots$ \\
\hline SB-1 & 32.58 & 37.56 & -90.58 & -32.39 \\
\hline SB-2 & 37.52 & 49.47 & -78.31 & -33.82 \\
\hline SB-3 & 42.53 & 51.87 & -72.81 & -34.71 \\
\hline
\end{tabular}

Table 3 shows that in the presence of SBs, the $\mathrm{H}^{*}$ values for mild steel dissolving in $1 \mathrm{M} \mathrm{HCl}$ are greater $\left(37.56-51.87 \mathrm{~kJ} \mathrm{~mol}^{-1}\right)$ than in the absence of inhibitors $\left(26.04 \mathrm{~kJ} \mathrm{~mol}^{-1}\right)$. The positive value of enthalpy indicates that the mild steel dissolution process is endothermic, implying that mild steel dissolution is more difficult in the presence of SBs than in the absence of SBs. When the values of entropy of activation $\left(\Delta S^{*}\right)$ in Table 3 are compared to the free acid solution, it is obvious that entropy of activation increased in the presence of the examined SBs [21-23]. The phenomenon of ordering and disordering of inhibitor molecules on the mild steel surface is linked to this fluctuation. The higher activation entropy in the presence of SBs suggested that on the metal/solution interface, disorder increases as you move from reactant to activated complex.

\subsection{Electromechanical measurements}

\subsubsection{Potentiodynamic polarization measurements}

To learn more about the kinetics of the cathodic and anodic processes, polarisation measurements were performed. The cathodic and anodic curves for three SBs at their optimal concentration in $1 \mathrm{M} \mathrm{HCl}$ are shown in Figure 4. With the addition of SBs, both the cathodic and anodic reactions were suppressed, implying that the SBs reduced anodic dissolution while simultaneously delaying the hydrogen evolution reaction. Table 4 lists the values of electrochemical parameters related to polarisation measurements, such as corrosion potential (Ecorr), corrosion current densities (Icorr), and Tafel slopes (a, c), which were derived by extrapolating the Tafel slope. The table shows that inhibition efficiency rises with increasing concentrations, with the highest efficiency of 96 percent found at $400 \mathrm{ppm}$. 


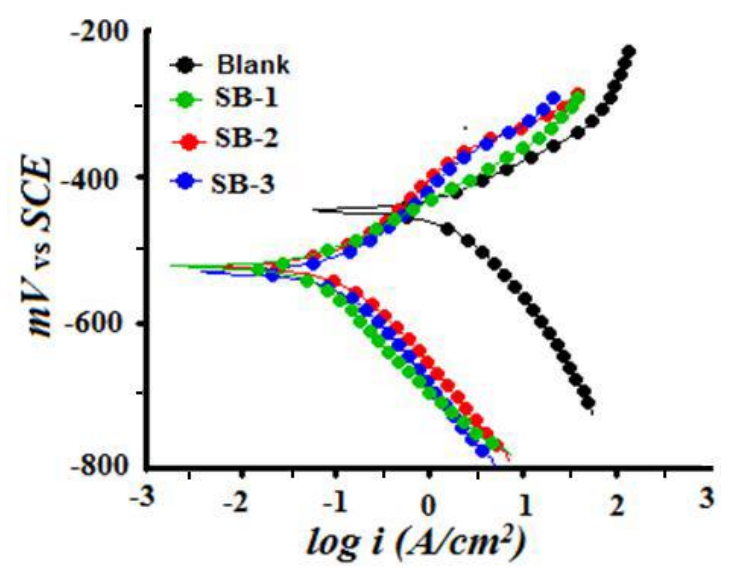

Figure 4 Tafel polarisation curves for mild steel corrosion in $1 \mathrm{M} \mathrm{HCl}$ in the presence and absence of optimal base concentrations.

According to Ferreira and others, if the (Ecorr) values are shifted:

(i) $>85 \mathrm{mV}$ in inhibited system with respect to uninhibited, the inhibitors could be recognized as cathodic or anodic type and

(ii) If displacement in Ecorr is $<85 \mathrm{mV}$, it could be recognized as mixed-type.

\subsubsection{Electromechanical impedance spectroscopy}

The impedance approach offers data on the kinetics of electrode processes as well as the surface properties of the systems being studied. Mechanistic information can be gleaned from the shape of impedance. After immersion for 30 minutes at $3031 \mathrm{~K}$, EIS evaluated the corrosion behaviour of mild steel in $1 \mathrm{M} \mathrm{HCl}$ in the absence and presence of different concentrations of SBs. Figures 4 (a) and (b) show Nyquist and Bode graphs of mild steel in uninhibited and inhibited acid solutions with optimum SB concentrations (b).
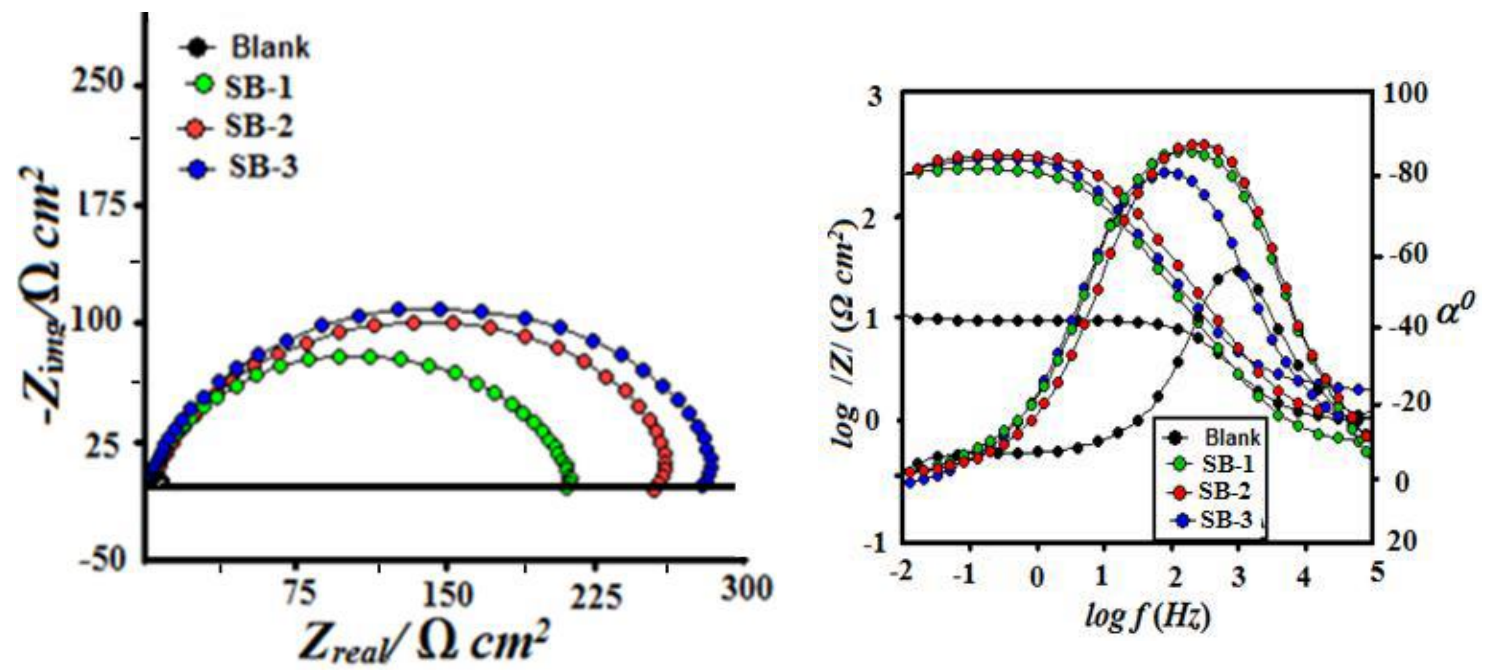

Figure 5 a) Nyquist plot in the absence and presence of optimal Schiff's bases concentrations b) Bode plot in the absence and presence of optimum Schiff's bases concentrations. 


\section{Surface investigation}

\subsection{SEM analysis}

Figure 6 (a-d) shows the morphology of mild steel specimens immersed in $1 \mathrm{M} \mathrm{HCl}$ for 3 hours in the absence and presence of the optimum SB concentration. Figure 6 (a) shows the surface morphology of unconstrained mild steel, which is characterised by a very rough surface with cracks and pits due to rapid corrosion attack; it can be concluded that the mild steel surface was severely damaged in the absence of SBs. The surface morphology of the inhibited mild steel specimens is given in Figure (b-d), and it can be concluded that in the presence of SBs, the mild steel surface morphology is significantly enhanced. This observation also points to the creation of a protective film of SBs on the surface of mild steel.
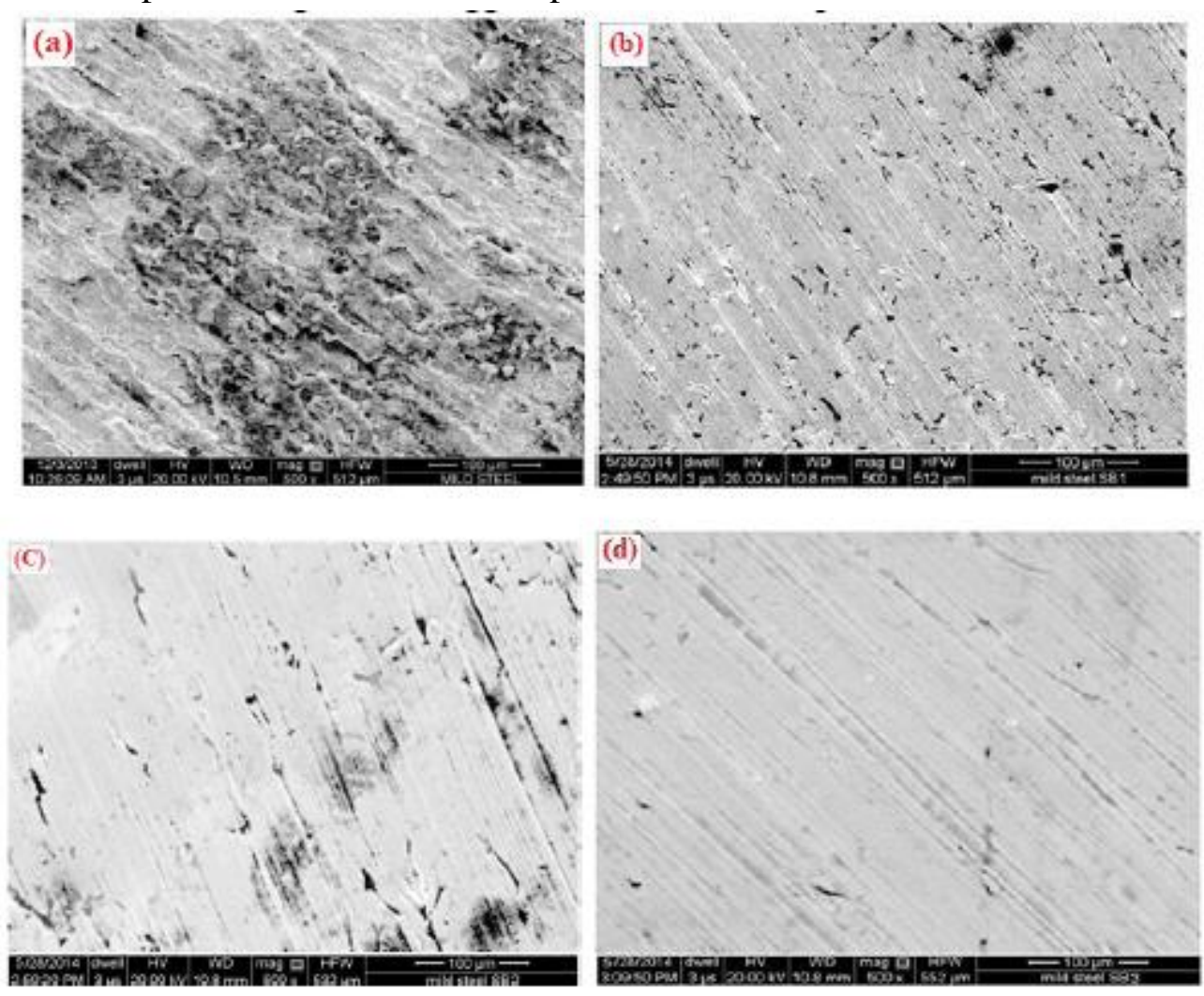

Figure 6 (a-d): SEM micrographs of mild steel surfaces

(a) in $1 \mathrm{M} \mathrm{HCl} \mathrm{(b)} \mathrm{in} \mathrm{presence} \mathrm{of} \mathrm{SB-1} \mathrm{(c)} \mathrm{in} \mathrm{presence} \mathrm{of} \mathrm{SB-2} \mathrm{(d)} \mathrm{in} \mathrm{presence} \mathrm{of} \mathrm{SB-3}$

\subsection{EDX Analysis}

The goal of this study was to confirm that SBs form a protective coating on mild steel surfaces based on weight loss and electrochemical studies. The EDX spectra of mild steel in $1 \mathrm{M} \mathrm{HCl}$ were recorded in the absence and presence of the optimum concentration of SBs to achieve this goal. 


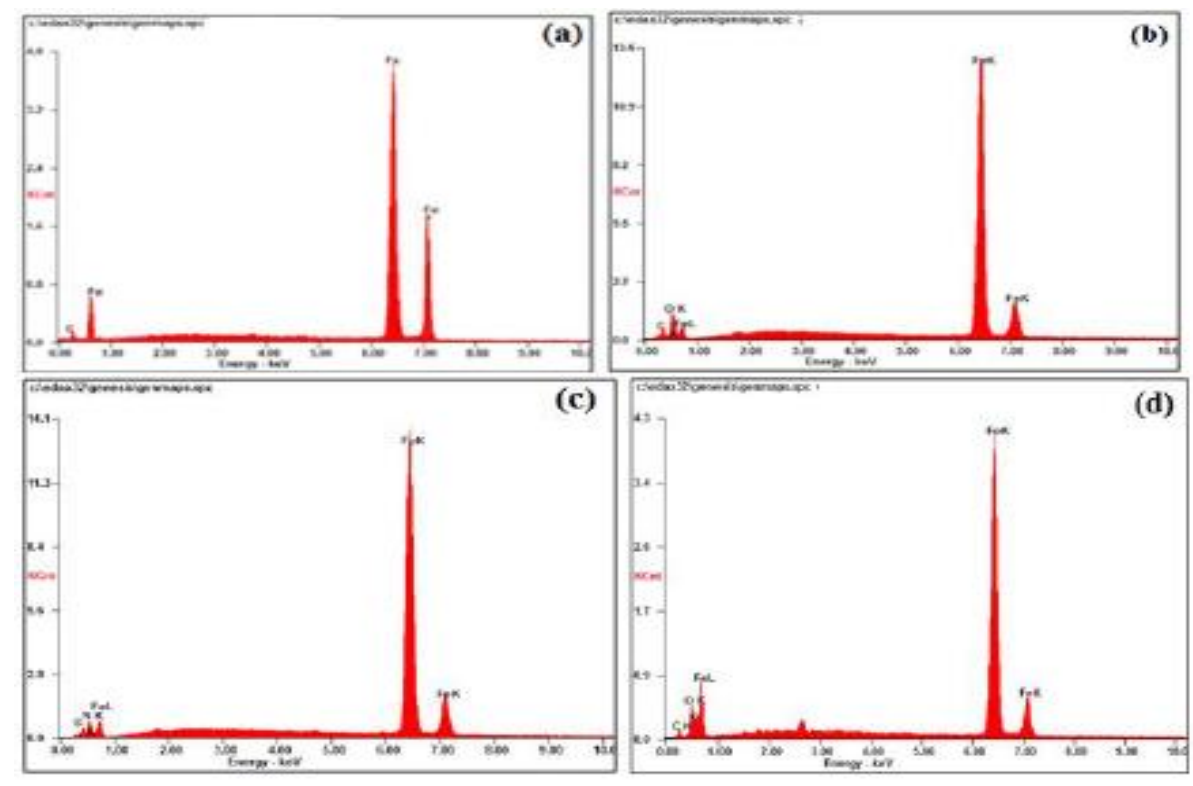

Figure 7 (a-d): EDX spectra of mild steel surfaces:

(a) in $1 \mathrm{M} \mathrm{HCl}(\mathrm{b})$ in presence of SB-1 (c) in presence of SB-2 (d) in presence of SB-3

Figure 7 (a-d) shows the EDX spectra of mild steel in the absence and presence of SBs, and table 6 shows the percentage atomic content determined from the EDX spectra. Figure 7 (a) shows the EDX spectra of unfettered mild steel with no peaks for O or N. However, in the presence of SBs, the EDX spectra of mild steel reveals distinct peaks for oxygen and nitrogen, implying that SBs create a protective coating on the mild steel surface.

Table 4: EDX spectra reveal the percentage atomic concentrations of elements on mild steel surfaces.

\begin{tabular}{|l|l|l|l|l|}
\hline Inhibitors & Fe & C & N & O \\
\hline- & 63.09 & 36.10 & - & - \\
\hline SB-1 & 65.57 & 26.36 & - & 8.02 \\
\hline SB-2 & 64.12 & 25.89 & 9.47 & - \\
\hline SB-3 & 62.65 & 24.28 & - & 12.87 \\
\hline
\end{tabular}

\section{Mechanism of inhibitions:}

SBs can adsorb on the mild steel surface in the following ways: (a) electrostatic interaction between charged molecules and charged metal; (b) interaction of -electrons with the metal; (c) interaction of the molecule's unshared pair of electrons with the metal; and (d) a combination of all the effects.

In the case of inhibitors, the effectiveness of inhibition is determined by a number of adsorption sites and their charge density, molecule size, hydrogenation heat, method of contact with the metal surface, and the creation of metallic complexes. SB inhibition efficiency is listed in the following order: SB-3 $>$ SB-2 $>$ SB-1.

Organic inhibitor adsorption cannot be considered merely physical or chemical phenomena. Nature, charge on metal surfaces, and inhibitor chemical structure all influence the adsorption mechanism. The electric field that arises at the metal/electrolyte interaction 
causes the charge on the metal surface. It is commonly known that mild steel specimens are positively charged in acid solutions when compared to the potential of zero charge (PZC). The fact that inhibitors not only supply of electrons to metal atoms but also have unoccupied higher energy orbitals to absorb electrons from the metal atom's dorbital for enhancing the bonding relationship is well recognised. Figure 8 shows a schematic representation of the many types of SB adsorption at the metal/acid contact.

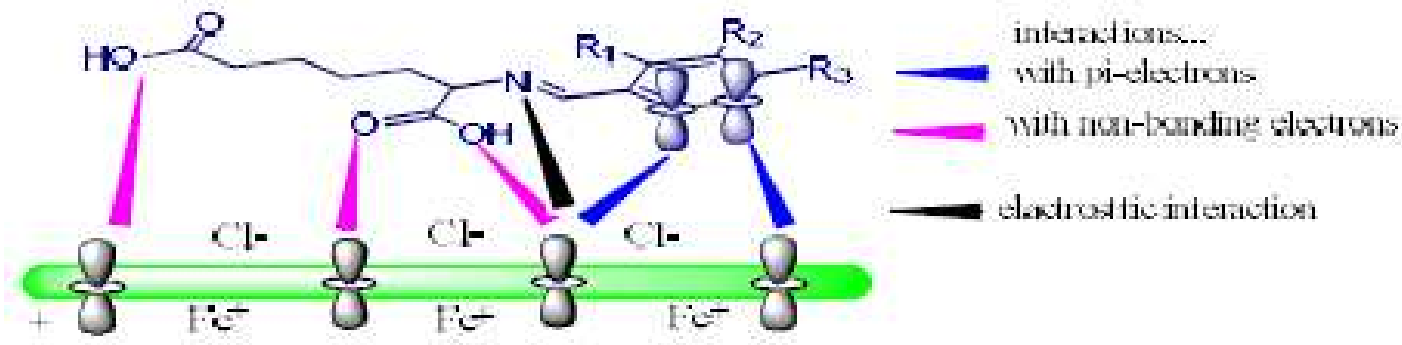

Figure 8 depicts the various forms of adsorption by SBs on a mild steel $1 \mathrm{M} \mathrm{HCl}$ interface.

\section{Conclusions}

The following conclusions can be drawn from the research: SBs are effective corrosion inhibitors for mild steel in a 1M HCL solution. At $400 \mathrm{ppm}$ concentration, the maximum efficiency was found to be 97 percent. The Langmuir isotherm was followed by the adsorption of SBs molecules on mild steel surfaces. SBs are mixed type inhibitors, meaning they impact both cathodic and anodic processes, according to potentiodynamic research. The fact that $G$ is negative indicates that inhibitor adsorption on mild steel is a spontaneous process. The outcomes of the weight-loss and electrochemical approaches are very similar.

\section{References}

1. H. Naeimi and A. Heidarnezhad, "Facile, mild and convenient preparation and characterization of some novel Schiff base ligands from synthetic diamines and salicylaldehyde," Bulletin of Chemical Society of Ethiopia, vol. 29, no. 1, pp. 117-122, 2015.View at: Publisher Site | Google Scholar

2. D. Zhu and W. J. V. Ooij, "Corrosion protection of AA 2024-T3 by bis-[3(triethoxysilyl) propyl]tetrasulfide in neutral sodium chloride solution. Part 1: Corrosion of AA 2024-T3," Corrosion Science, vol. 45, no. 10, pp. 2163-2175, 2013.View at: Google Scholar

3. T. G. Harvey, S. G. Hardin, A. E. Hughes et al., "The effect of inhibitor structure on the corrosion of AA2024 and AA7075," Corrosion Science, vol. 53, no. 6, pp. 2184-2190, 2011.View at: Publisher Site | Google Scholar

4. "Standard Practice for Laboratory Immersion Corrosion Testing of Metal-G31-722004".View at: Google Scholar

5. W. H. Li, Q. He, S. T. Zhang, C. L. Pei, and B. R. Hou, "Some new triazole derivatives as inhibitors for mild steel corrosion in acidic medium," Journal of Applied Electrochemistry, vol. 38, no. 3, pp. 289-295, 2008. View at: Publisher Site | Google Scholar

6. Yurt, S. Ulutas, and H. Dal, "Electrochemical and theoretical investigation on the corrosion of aluminium in acidic solution containing some Schiff bases," Applied Surface Science, vol. 253, no. 2, pp. 919-925, 2006.View at: Publisher Site | Google Scholar 
7. M. G. Fontana, Corrosion Engineering, McGraw-Hill, New York, Third Edition edition, 1987.

8. H. C. A. Murthy and S. K. Singh, "Influence of TiC particulate reinforcement on the corrosion behaviour of Al 6061 metal matrix composites," Advanced Materials Letters, vol. 6, no. 7, pp. 633-640, 2015.View at: Google Scholar

9. E. A. Matter, S. Kozhukharov, M. Machkova, and V. Kozhukharov, "Electrochemical studies on the corrosion inhibition of AA2024 aluminium alloy by rare earth ammonium nitrates in 3.5\% NaCl solutions," Materials and Corrosion, vol. 64, no. 5, pp. 408-414, 2013.View at: Publisher Site | Google Scholar

10. D. Ho, N. Brack, J. Scully, T. Markley, M. Forsyth, and B. Hinton, "Cerium dibutylphosphate as a corrosion inhibitor for AA2024-T3 Aluminum alloys," Journal of the Electrochemical Society, vol. 153, no. 9, pp. B392-B401, 2006.View at: Publisher Site | Google Scholar

11. M. G. Fontana and N. D. Greene, Corrosion Engineering, McGraw-Hill, New York, 1967.

12. F. N. Spellar, Corrosion: Causes and Prevention- an Engineering Problem, Mc Graw Hill, New York, 8 Edition edition, 1935.

13. H. C. Ananda Murthy, "Electroanalytical study on the corrosion behaviour of $\mathrm{TiO} 2$ particulate reinforced Al 6061 composites," Material Science Research India, vol. 12, no. 2, pp. 112-126, 2015.View at: Publisher Site | Google Scholar

14. W. H. Ailor, Engine coolant testing: state of the art, ASTM International, 1980.

15. H. A. Murthy, V. B. Raju, and C. Shivakumara, "Effect of TiN particulate reinforcement on corrosive behaviour of aluminium 6061 composites in chloride medium," Meddelelser fra Carlsberg laboratoriet, vol. 36, no. 6, pp. 1057-1066, 2013.View at: Publisher Site | Google Scholar

16. J. A. V. Butler, "Studies in heterogeneous equilibria. Part II.-The kinetic interpretation of the Nernst theory of electromotive force," Trans. Faraday Soc., vol. 19, no. March, pp. 729-733, 1924.View at: Publisher Site | Google Scholar

17. H. J. W. Lenderink, M. V. D. Linden, and J. H. W. De Wit, "Corrosion of aluminium in acidic and neutral solutions," Electrochimica Acta, vol. 38, no. 14, pp. 1989-1992, 1993.View at: Publisher Site | Google Scholar

18. F. Mansfeld, C. H. Tsai, and H. Shih, Computer Modelling in Corrosion, R. S. Munn, Ed., ASTM, Philadelphia, PA, 1992.

19. O. Olivares, N. V. Lihanova, B. Gomez et al., "Electrochemical and XPS studies of decylamides of $\alpha$-amino acids adsorption on carbon steel in acidic environment," Applied Surface Science, vol. 252, no. 8, pp. 2894-2909, 2006.View at: Publisher Site | Google Scholar

20. M. Pourbiax, Atlas of Electrochemical Equilibria in Aqueous Solutions, NACE International, Houston, TX, 1974.

21. M. Iannuzzi and G. S. Frankel, "Mechanisms of corrosion inhibition of AA2024-T3 by vanadates," Corrosion Science, vol. 49, no. 5, pp. 2371-2391, 2007.View at: Google Scholar

22. H. Elgahawi, M. Gobara, and A. Baraka, "Eco-friendly corrosion inhibition of AA2024 in 3.5\% NaCl using the extract of Linum usitatissimum Seeds," Journal of Bio and Tribo Corrosion, vol. 3, no. 4, pp. 55-67, 2017.View at: Google Scholar

23. E. S. Ferreira, C. Giacomelli, F. C. Giacomelli, and A. Spinelli, "Evaluation of the inhibitor effect of L-ascorbic acid on the corrosion of mild steel," Materials Chemistry and Physics, vol. 83, pp. 129-134, 2004. View at: Google Scholar. 